\title{
Transplanting a Black Kidney from a Albino - First Case Report
}

Aditya Avinash Yelikar* and Sachin Joseph

Department of Urology, Medical Trust Hospital, Kerala, India

*Corresponding author: Aditya Avinash Yelikar, 3rd year DNB Trainee, Department of Urology, Medical Trust Hospital, M.G. Road, Kochi - 682020, Kerala, India, Tel: +91 9823733333; E-mail: aditya.yelikar@gmail.com

Received date: June 12, 2017; Accepted date: June 21, 2017; Published date: June 28, 2017

Copyright: (c) 2017 Yelikar AA, et al. This is an open-access article distributed under the terms of the Creative Commons Attribution License, which permits unrestricted use, distribution and reproduction in any medium, provided the original author and source are credited.

\begin{abstract}
Our case report is the only reported case in literature of transplanting a black coloured kidney successfully from a albino donor. Oculocutaneous albinism is similar to hermansky-pudlak syndrome and chediak higashi syndrome and could lead to more widespread lysosome excretory defects. These defects could lead to accumulation of some intra cellular material, leading to the gross discolouration of the kidney. Hermansky pudlak syndrome is a triad of albinism, platelets lacking dense bodies and storage of ceroid like material in tissues. The manifestations of storage disease are variable and include granulomatous colitis, restrictive lung disease, kidney failure and cardiomyopathy. The autofluorescent material stored in the hermansky pudlak syndrome contains dolichols. Our patient was a living related donor who was having oculocutaneous albinism. Her kidneys were normal functioning but on exposure was black coloured. Post transplant the graft showed ATN and parenchymal dysfunction which later recovered.
\end{abstract}

Keywords: Kidney; Albinism; Transplant

\section{Abbreviations:}

HPS: Hermansky-Pudlak syndrome; CHS: Chediak Higashi syndrome

\section{Introduction}

Oculocutaneous albinism is similar to hermansky -pudlak syndrome and chediak higashi syndrome and could lead to more widespread lysosome excretory defects [1]. These defects could lead to accumulation of some intra cellular material, leading to the gross discolouration of the kidney. Hermansky pudlak syndrome is a triad of albinism, platelets lacking dense bodies and storage of ceroid like material in tissues [1]. The manifestations of storage disease are variable and include granulomatous colitis, restrictive lung disease, kidney failure and cardiomyopathy. The auto-fluorescent material stored in the hermansky pudlak syndrome contains dolichols [2]. Our patient was a living related donor who was having oculocutaneous albinism. Her kidneys were normal functioning but on exposure was black coloured (Figure 1). Post transplant the graft showed ATN and severe parenchymal dysfunction which later recovered.

\section{Case Presentation}

Our patient was a 36 years old female with occulocutaneous albinism who was admitted for donor nephrectomy to donate kidney to her brother who was suffering from ESRD and ocular albinism. She was investigated for same in the routine manner.

Usg - Endometrial polyp 1.6 by $1.7 \mathrm{~cm}$

$24 \mathrm{hr}$ Urine protein -86.4

Urine culture and $\mathrm{R} / \mathrm{E}$ were normal

HDL Cholesterol 54

LDL Cholesterol 114 cholesterol 184 total bilirubin 0.3 direct 0.1
SGOT 12 SGPT 28 ALP 99 protein 7.7 albumin 3.8 creatinine 0.9

Haemoglobin 11.3 PLT 306000

DTPA - LK GFR - $55 \mathrm{ml} / \mathrm{min} 49 \%$ relative uptake

Peak to half peak time 9.5

Since all her investigations were normal we posted her for open left donor nephrectomy. On exposure the kidney was found to be black coloured (Figure 1).

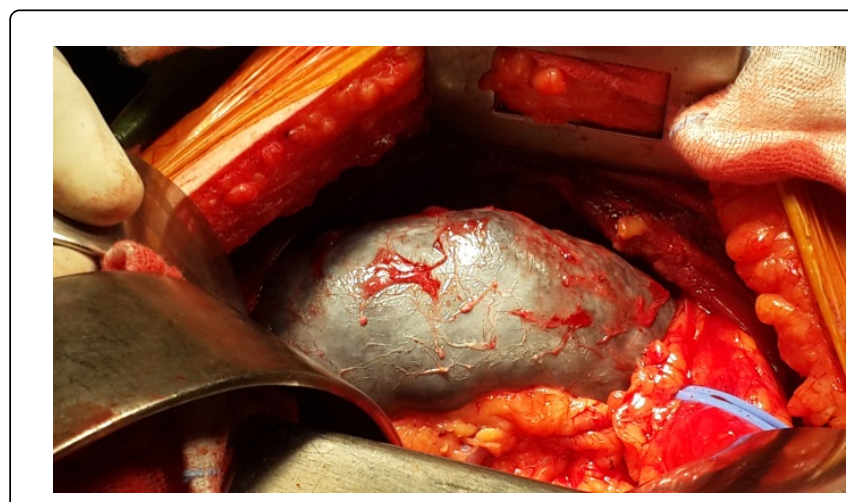

Figure 1: Post exposure while open donor nephrectomy the kidney was black coloured.

After taking opinions from the nephrologist, colleague urologist, anaesthetists we decided to proceed with the surgery since there was no case report in the literature suggesting any contraindication for transplanting such a black coloured normal functioning kidney. We harvested the graft and placed it in the right iliac fossa of the recipient followed by usual vascular anastomosis. There was adequate urine output from the graft kidney intraoperatively. After 10-12 hours recipient's output started declining from $250 \mathrm{ml} /$ hour to $100 \mathrm{ml} /$ hour. His creatinine also raised from 4.3 to 5.5 . Renal biopsy was done which was suggestive of acute tubular necrosis with tubular epithelial cells 
showing prominent cytoplasmic brownish granular pigment deposits (Figure 2).

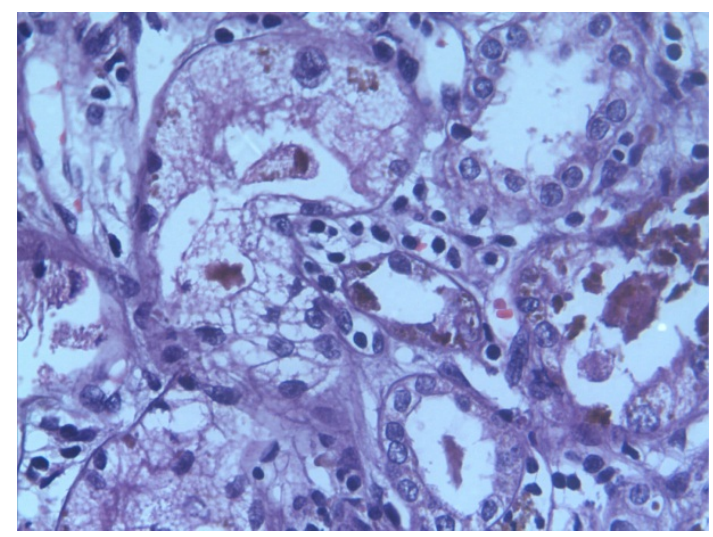

Figure 2: Immediate post transplant biopsy pathology slide showing intra-cytoplasmic deposition of brownish granular pigment.

This decline in the function of transplanted kidney continued and on 9th post op day his creatinine was 9. The donor's post op period was uneventful. Her creatinine on discharge was 1.3. On post op day 20 we did a DTPA renal scintigraphy of recipient which showed that the graft kidney was having a GFR of $8.97 \mathrm{ml} / \mathrm{min}$, time to peak $3.07 \mathrm{mins}$. It showed severe parenchymal dysfunction of transplant kidney. He underwent multiple HD sessions. After 6 weeks the graft function started improving showed by reducing trend of serum creatinine and at the end of 9 weeks on follow up his creatinine was 1.6.

\section{Discussion}

To our knowledge this is the first case report in literature of transplanting a black coloured kidney of an occulocutaneous albino to an ocular albino. HPS causes abnormalities in the biosynthesis and function of melanosomes, platelets, immune cells, lysosomal excretory defects and storage of ceroid like material in kidneys, heart and lungs [1]. Ceroid contains dolichols, levels of which are elevated in urine, in those patients with evidence of ceroid storage in the kidneys but are not elevated when storage occurs in tissues other than kidneys [2]. There is also an elevation of kidney beta galactosidase due to defective urinary excretion of lysosomal enzymes [3]. CHS is characterized by recurrent infections and death in midlife [1]. HPS is characterized by frequent bleeding diathesis [1]. Renal hemosiderosis which is a known complication of chronic intravascular hemolysis can cause black discolouration of kidney [1]. Our patient had a smooth post op period after the donor nephrectomy but the recipient did not do well initially. After transplanting the graft in the recipient he had a rising trend of serum creatinine. Colour Doppler of the transplant kidney was normal with RI of 0.7. Biopsy from the graft showed ATN and cytoplasmic deposits of brownish granular pigment. This pigment was PAS negative. So it is unlikely that this material is similar to that stored in neuronal ceroid/lipofuscinosis which is PAS positive. He underwent hemodialysis twice weekly and had an average output of 1.2 lit/day. Though the graft was viable it had severe parenchymal dysfunction as showed by the DTPA renogram. In our case findings not favouring HPS were she had normal platelets, no bleeding diathesis and the ceroid material was PAS negative. Findings not favouring CHS were her age (CHS presents in pediatric age group) and she never had any major infections in past. Findings not favouring Renal Hemosiderosis were she had no clinical or laboratory findings of hemosiderosis. Urinary dolichol levels could not be assesed in our patient due to nonavailability of this test. In our case the findings in favour of HPS were presence of oculocutaneous albinism and black coloured kidney.

\section{Conclusion}

Further research is needed to find out the causes of black coloured kidney other than HPS and CHS in oculocutaneous albinos.

Urinary dolichol levels should be done in all cases of occulocutaneous albinism before donor nephrectomy to rule out HPS.

Possibility of ATN/graft dysfunction should be kept in mind before transplanting a black coloured kidney from an oculocutaneous albino. Such kidneys when transplanted may show a delayed improvement in graft function.

More research in needed to find out factors causing graft dysfunction and factors preventing it for transplanting such kidney.

We do not know what would have been the outcome if such kidney was transplanted in a normal person.

Role of laparoscopic donor nephrectomy should be considered in reducing donor morbidity in cases where the nephrectomy is to be abandoned on coming across a black coloured kidney.

\section{References}

1. Huizing M, Anikster Y, Gahl WA (2000) Hermansky-Pudlak syndrome and related disorders of organelle formation. Traffic 1: 823-835.

2. Witkop CJ, Wolfe LS, Cal SX, White JG, Townsend D, et al. (1987) Elevated urinary dolichol excretion in the Hermansky - Pudlak syndrome. Indicator of lysosomal dysfunction. Am J Med 82: 463-470.

3. Meisler MH (1978) Synthesis and secretion of kidney beta-galactosidase in mutant le/le mice. J Biol Chem 253: 3129-3134. 